INF. \& ANN.

\title{
National Instructional Workshop in Cryptology 6-10 May 2013, Cochin
}

\author{
Sponsored by \\ Cryptology Research Society of India, Organised by Department of Mathematics, \\ Cochin University of Science and Technology
}

Call for Participation: This workshop is aimed to familiarize the subject of Cryptology and to motivate young students to opt a research career in this and related subjects.

Eligibilty: PG students of Mathematics /Engineering, Research Scholars, Teachers with less than 6 years experience in any recognized institution in India. Exceptionally good BTech. students can also participate.

Topics for discussion: Number theoretic background, Introduction to cryptography, Symmetric Key cryptography, Elliptic curve cryptography, Computational issues in cryptography, Cryptographic Hash Functions, Provably Secure public key encryption and digital signature.

Resource Persons: R Balasubramanian (IMSc., Chennai), Abhijit Das(IIT Kharagpur), Somitra Kumar Sanadhya (IIIT, Delhi), Sourav Sengupta ( ISI, Kolkata), Somindu C Ramanna (ISI, Kolkata).

Travel support: All selected participants are eligible for reimbursement up to 3 AC.

For further details contact : Prof. Ambat Vijayakumar, Coordinator, NIWS in Cryptology, Department of Mathematics, CUSAT, Cochin-682 022. Email: vambat@gmail.com

RESONANCE | February 2013 